\title{
La oportunidad de incorporar el bienestar subjetivo en la protección de la infancia y adolescencia en Chile
}

\author{
The opportunity to incorporate subjective well-being in the protection \\ of children and adolescents in Chile
}

\author{
Baltica Cabieses $^{\mathrm{a}}$, Alexandra Obach $^{\mathrm{a}}$, Xaviera Molina ${ }^{\mathrm{a}}$
}

aPrograma en Estudios Sociales en Salud, ICIM, Facultad de Medicina Clínica Alemana, Universidad del Desarrollo, Chile

Recibido: 26 de noviembre de 2019; Aceptado: 16 de enero de 2020

¿Qué se sabe del tema que trata este estudio?

La situación de vulnerabilidad en niños, niñas y adolescentes en Chile genera efectos que tienen un impacto en todo el curso de vida. Una forma de evaluar la calidad de vida de esta población, afectada por situaciones de vulnerabilidad, es el indicador de bienestar subjetivo.

\begin{abstract}
¿Qué aporta este estudio a lo ya conocido?
El artículo aporta proponiendo el uso del índice de bienestar subjetivo en las políticas pública para niños, niñas y adolescentes, como una herramienta que promueve la protección de la salud y calidad de vida de esta población en Chile.
\end{abstract}

\section{Resumen}

Los niños, niñas y adolescentes (NNA) representan a un cuarto de la población chilena, sobre la cual en términos de protección el país se ha enfocado en la promoción de igualdad de oportunidad de derechos en base a convenciones internacionales. Sin embargo, aún los NNA siguen muriendo por causas prevenibles y modificables, además de experimentar importantes grados de vulnerabilidad, situación que repercute en complejos efectos emocionales y cognitivos a lo largo de su ciclo vital. Según la evidencia internacional el bienestar de los NNA va directamente relacionado con los índices de desigualdad social, valorando el bienestar como un componente esencial y transversal a los derechos de los NNA del país. En Chile, el bienestar subjetivo se define como las percepciones de los NNA respecto de sus condiciones vitales -tanto en el aspecto económico, como social, cultural, de salud y logros personales. Este concepto se ha estudiado a través de diferentes instrumentos los cuales podrían ser de gran utilidad para promover el componente psicosocial y calidad de vida de los NNA en las estrategias y políticas nacionales en esta población. En esta revision se propone integrar de manera explícita en las políticas públicas de la infancia y adolescencia el concepto de bienestar subjetivo, con el fin de entregar oportunidades justas, positivas y sistemáticas para el máximo desarrollo de los NNA en su ciclo vital, validando empíricamente las condiciones necesarias del buen vivir basado en un enfoque moderno de derechos humanos al cual el país se ha comprometido.
Palabras clave:

Bienestar subjetivo; infancia; políticas públicas; desigualdad

xmolinaa@udd.cl 


\begin{abstract}
Children and adolescents (CA) represent a quarter of the Chilean population, on which the country, regarding protection, has focused on the promotion of equal rights opportunities based on different international conventions. However, CA continue to die from preventable and modifiable causes in addition to experiencing severe degrees of vulnerability, a situation that has an impact on complex emotional and cognitive effects through their life cycle. According to international evidence, the welfare of CA is directly related to social inequality indices, valuing well-being as an essential and transversal component of the rights of CA in the country. In Chile, subjective well-being is defined as the perceptions of CA regarding their living conditions, both economic, social, cultural, and health aspects and personal achievements. This concept has been studied through different instruments which could be very useful to promote the psychosocial component and quality of life of CA in national strategies and policies in this population. This review proposes to explicitly integrate into the public policies of CA the concept of subjective well-being in order to provide fair, positive and systematic opportunities for the maximum development of CA in their life cycle, empirically validating the necessary conditions for good living based on a modern human rights approach that the country has committed to.
\end{abstract}

\section{Keywords:}

Subjective well-being;

childhood;

public policies;

inequality
El mundo está cambiando rápidamente. Donde había alrededor de 5 mil millones de personas en 1990, para el 2050 habrá cerca de 10 mil millones, de los cuales más de 2.6 mil millones de ellos son menores de 18 años. En Chile, en el año 2017 se estimó que existen 4.259.155 niñas, niños y adolescentes (NNA), es decir, casi un cuarto de la población nacional ${ }^{1}$. En términos de protección de la infancia y adolescencia, el país se ha enfocado en la promoción de igualdad de oportunidad de derechos, de acuerdo con lo establecido en los principios de la Convención sobre los Derechos del Niño del $1990^{2}$, la cual busca brindar la oportunidad de crecer sanos, educados y capaces de desarrollar todo su potencial y convertirse en ciudadanos plenamente participantes. Esta convención potencia la identidad y los derechos propios de NNA, con el fin de alcanzar el reconocimiento de sus propios derechos humanos. En concordancia con esto, el Estado de Chile ha ratificado su adscripción a las convenciones internacionales en materia de bien superior de la infancia y adolescencia. No obstante, se reconoce que se debe continuar con esfuerzos en esta materia para extender el alcance y propósito de las políticas y acciones existentes en la actualidad para NNA. Solo así se podrá garantizar el desarrollo sostenido de un enfoque de derecho y de bienestar infantil en todos los ámbitos de desarrollo humano en nuestro país.

A nivel mundial, la situación de algunos grupos de NNA resulta alarmante. De acuerdo con el reporte del 2015 de mortalidad infantil de UNICEF$^{3}, 16.000$ niños y niñas mueren cada día. La mayoría de estas muertes provienen de países en vías de desarrollo y por causas que son prevenibles y modificables, como enfermedades infecciosas secundarias por falta de agua potable y tratamiento médico básico. Por otro lado, de aque- llos que sobreviven, cientos de millones experimentan grados preocupantes de vulnerabilidad social, económica y política. Esta vulnerabilidad está asociada, por ejemplo, a situación de pobreza material absoluta, baja escolaridad, abandono, migración informal, exclusión social por pertenecer a grupos sociales marginados, entre otros ${ }^{4}$. En el escenario nacional, pese a existir una ley que prohíbe el trabajo infantil, según la Encuesta Nacional de Actividades de Niños, Niñas y Adolescentes del $2012^{5}$ un 6,6\% niños chilenos entre 6 a 17 años estarían en situación de trabajo infantil, y el 10\% de este grupo lo hacen con menos de 14 años. En lo relativo a las cifras de escolaridad, se observa que un $10 \%$ de niños menores de 14 años no asisten a educación básica. A esto se le suma la situación de pobreza y condiciones de vida que experimentan ciertos grupos de NNA en nuestro país, en donde uno de cada diez vive en situación de pobreza absoluta por ingresos, cifra que se duplica en el caso de NNA indígenas y migrantes ${ }^{1}$.

Las consecuencias que diversas situaciones sociales de vulnerabilidad provocan en NNA son cualitativamente diferentes a las de los adultos, ya que estos tienen necesidades específicas que, si no se satisfacen, generan limitaciones que impactan en su desarrollo y bienestar futuro. Hallazgos extraídos de diversos estudios internacionales de los últimos 20 años ${ }^{6}$ proponen desde neurobiología del estrés que, en ausencia de atención y apoyo, los estresores experimentados durante períodos sensibles del desarrollo pueden dejar huellas permanentes a nivel neuronal en los procesos emocionales y cognitivos. Estas huellas pueden generar, por ejemplo, severos trastornos crónicos cognitivos, especialmente cuando se combinan con entornos adversos y factores estresantes múltiples ${ }^{4,6}$. La evidencia internacional propone que un NNA que no tiene un acceso tempra- 
no a una buena educación y/o nutrición, sobre todo en escenarios socioeconómicos y familiares de carencia y abuso, podría tener consecuencias negativas visibles, tanto en lo físico como en lo intelectual y emocional ${ }^{7,8}$, las que serían difíciles de modificar. Como también existirían diversos mecanismos que podrían explicar las influencias de situaciones sociales de vulnerabilidad en edad temprana en resultados de salud a lo largo de la vida. Por ejemplo, se ha encontrado asociación entre estrés tóxico en etapa intrauterina y enfermedades crónicas ${ }^{9}$, así como también asociación entre privación socioeconómica ${ }^{10}$ y problemas de apego, vínculo y regulación emocional en la adultez ${ }^{11}$. De esta forma, diversas hipótesis han ido probando su posible fuerza de relación causal entre situaciones sociales de vulne- rabilidad y riesgos en salud. Una selección de estudios internacionales relevantes en esta temática se presenta en la tabla 1.

Estas problemáticas se originan, en gran medida, a partir de la desigualdad social que los países enfrentan, especialmente en las áreas de ingreso y educación. En el caso de Chile está demostrada en la Política Nacional de Niñez y Adolescencia 2015-2025 ${ }^{12}$ al indicar que "los efectos negativos de esta desigual distribución del ingreso son múltiples, tanto a nivel de integración y cohesión social como en las diferencias de las oportunidades a las que acceden los ciudadanos, incluyendo por cierto a los niños, niñas y adolescentes. Así, la desigualdad económica se traduce en inequidades que se confirman en el ámbito de la educación, salud, vivienda, barrios, entre otros". El

\section{Tabla complementaria 1. Principales hallazgos de la evidencia internacional de la neurobiología del estrés}

\begin{tabular}{llc}
\hline Nombre de la revisión & Autores & Población \\
\hline $\begin{array}{l}\text { Principales hallazgos } \\
\text { desde la neuropsicología } \\
\text { del estrés }\end{array}$ & $\begin{array}{c}\text { Hair NL, } \\
\text { Hanson JL, } \\
\text { Wolfe BL, } \\
\text { Pollak SD. }\end{array}$ & \\
& \\
& \\
& & \\
& & \\
Toxic Stress: Effects, & Hillary AF. & Niños y niñas \\
$\begin{array}{l}\text { Prevention and } \\
\text { Treatment }\end{array}$ & Franke &
\end{tabular}
Principales hallazgos desde la neuropsicología del estrés Los niveles de pobreza en NNA se asocia a menores habilidades escolares, lo cual se refleja en la estructura del desarrollo cerebral, se observa que en este grupo hay una disminución promedio entre 3 a 4 puntos porcentuales de la materia gris promedio de un NNA. Esta diferencia se acentúa aún más en los niveles de pobreza más crítico. Esta situación en los NN, se evidenció además en las consecuencias del rendimiento académico, obteniendo de 4 a 7 puntos menos en las pruebas estandarizadas. Alrededor del $20 \%$ de la brecha en los puntajes de las pruebas podría explicarse por los retrasos de maduración en los lóbulos frontal y temporal.

Prevention and

Franke

Se ha observado que uno de los efectos del estrés, en NN repercuten la salud a corto y largo plazo; que resultan en problemas de la salud como alcoholismo, enfermedad pulmonar obstructiva crónica, depresión, cáncer, obesidad, cardiopatía isquémica y muchos otros procesos de la enfermedad. Las estrategias de prevención y protección de los niños contra el estrés tóxico requieren intervenciones ambientales dirigidas también al cuidador y al medio ambiente. Por ejemplo, reducción del estrés, técnicas de afrontamiento y el uso de un enfoque integrador de la terapia.

The Impact of Childhood Maltreatment: A Review of Neurobiological and Genetic Factors
McCrory E, De Brito SA, Viding $\mathrm{E}$.

Arthur J et al. Niños y niñas

Effects of a SchoolBased, Early Childhood Intervention on Adult Health and Well-being

Early life stress as a risk factor for mental health: Role of neurotrophins from rodents to nonhuman primates
Cirulli F et al. Animales (roedores)
El maltrato infantil representa un factor de riesgo significativo para la psicopatología, afecta a el desarrollo psicológico y emocional de un niño. A nivel endocrino, demuestra una asociación entre el maltrato y el desarrollo atípico de la respuesta al estrés del eje hipotalámico-hipofisario-suprarrenal, lo que puede predisponer a la vulnerabilidad psiquiátrica en la edad adulta, esto se ha evidenciado técnicamente a través de las imágenes cerebrales por resonancia magnética.

En este estudio se realizó una intervención educativa padre e hijo/a preescolares pertenecientes a un nivel socioeconómico bajo. Los resultados evidenciaron una amplia gama de resultados positivos a través de la aplicación de un programa escolar, generando menores tasas de deserción escolar, menor número de delitos graves, condenas o encarcelamiento, además de menores síntomas depresivos y mejores logros educativos.

Los eventos adversos en los primeros años pueden conducir a una mayor susceptibilidad a la psicopatología en la edad adulta. Los factores neurotróficos, se ven afectados por el estrés y juegan un papel importante en el desarrollo del cerebro y en el trofismo de redes neuronales específicas involucradas en la función cognitiva y en los trastornos del estado de ánimo que pueden verse modificada por agentes estresores del sistema nervioso. Además, la activación desadaptativa o repetida de estos agentes, a temprana edad, puede influir en la sensibilidad al estrés en la edad adulta y aumentar la vulnerabilidad.

Elaboración propia. 
reciente informe del PNUD sobre Desarrollo Humano ${ }^{13}$, establece que Chile clasificó entre los países con un Índice de Desarrollo Humano (IDH) "muy alto", ocupando el lugar 42 (valor de 0.832 ), dentro del total de 188 países considerados. Sin embargo, al considerar el IDH ajustado por desigualdad, Chile retrocede 13 posiciones (hasta el valor 0,672 ), siendo superado en Latinoamérica solo por Uruguay y Argentina en este ajuste. Esto da cuenta de la importancia de la desigualdad socioeconómica en el desarrollo humano de las naciones, particularmente en nuestro país, e invita al reforzamiento de la protección de los derechos de NNA como una tarea que requiere de constante revisión y mejora, pese a los enormes esfuerzos que en esta materia se han realizado en el país en las últimas décadas (tabla 2). Así lo ha reconocido UNICEF en sus reportes mundiales más recientes ${ }^{14}$, al señalar que se debe: “... promover los derechos y el bienestar de los niños en todas partes y oportunidades justas en la vida para todos los niños, especialmente para los niños a los que con frecuencia se les niega esta oportunidad con el fin de asegurarse de que todos los niños - comenzando con los más desfavorecidos - tengan la oportunidad de sobrevivir, prosperar y alcanzar su máximo potencial “.

De acuerdo a lo señalado anteriormente por UNICEF, además de considerar los derechos de NNA, se propone potenciar el bienestar de NNA, definido como "el conjunto de percepciones, evaluaciones y aspiraciones de niños con respecto a sus propias vidas". El reporte de esta institución del año 2017 midió el bienestar en cinco dimensiones: Seguridad, recursos, educación, familiar y bienestar subjetivo. En este documento se sostiene que Reino Unido, Estados Unidos y Hungría presentan el mejor ranking total de bienestar en países de mayores ingresos. Sin embargo, este índice no se genera de manera aislada, sino que tiene relación directa con otros factores. De acuerdo a los informes de "The Equity Trust" -reconocida organización británica que trabaja en la difusión de investigaciones contra la inequidad social y económica, y que dedica especial atención a la situación determinante de los NNA-, a mayor inequidad en la distribución de ingresos en los países, menor será el ranking de bienestar infantil ${ }^{15}$. Así, países con menor inequidad de ingresos como, por ejemplo, Suiza, Holanda y Finlandia tienen mejores índices totales de bienestar en NNA. Ante esta evidencia se considera primordial el trabajo simultáneo de aumento del bienestar en este grupo etario, y la desigualdad socioeconómica de los países, para impactar de manera concreta en la salud en la infancia y adolescencia. De esta forma, el concepto bienestar de NNA se articula en un eje central de trabajo intersectorial en áreas del desarrollo humano, justicia social y protección de los más vulnerables.

Tabla 2. Estrategias involucradas con el bienestar infantil en Chile

\begin{tabular}{|c|c|c|c|}
\hline Nombre programa & Fecha inicio & Institución dependiente & Objetivo \\
\hline Espacios amigables & 2008 & $\begin{array}{l}\text { Ministerio de Salud, } \\
\text { División de Control } \\
\text { y Prevención de } \\
\text { Enfermedades }\end{array}$ & $\begin{array}{l}\text { Mejorar el acceso a la atención de Salud Integral, a fin de detectar e } \\
\text { intervenir oportunamente factores y conductas de riesgo, fortalecer con- } \\
\text { ductas protectoras, contribuir a disminuir el embarazo no planificado, } \\
\text { infecciones de trasmisión sexual, suicidio y otros problemas de salud, y } \\
\text { además contribuir a potenciar un desarrollo y crecimiento saludable. }\end{array}$ \\
\hline Chile Crece Contigo & 2007 & $\begin{array}{l}\text { Ministerio de Desarrollo } \\
\text { Social }\end{array}$ & $\begin{array}{l}\text { Acompañar, proteger y apoyar a todos los niños, niñas y sus familias, } \\
\text { a través de un sistema integrado de intervenciones sociales de carácter } \\
\text { universal y otras prestaciones diferenciadas para menores en situación } \\
\text { de vulnerabilidad de la gestación hasta los } 9 \text { años. }\end{array}$ \\
\hline $\begin{array}{l}\text { Programa Nacional de } \\
\text { Inmunización }\end{array}$ & 1978 & Ministerio de Salud & $\begin{array}{l}\text { Proteger a la población residente en Chile, frente a enfermedades inmu- } \\
\text { noprevenibles relevantes para la salud pública, con calidad, seguridad y } \\
\text { eficiencia, acorde al desarrollo biotecnológico y la evidencia científica. }\end{array}$ \\
\hline JUNJ & 1970 & Ministerio de Educación & $\begin{array}{l}\text { Entregar Educación Parvularia de calidad y bienestar integral a niños y } \\
\text { niñas preferentemente entre } 0 \text { y } 4 \text { años de edad, priorizando las familias } \\
\text { con mayor vulnerabilidad socioeconómica, a través de una oferta pro- } \\
\text { gramática diversa y pertinente a los contextos territoriales. }\end{array}$ \\
\hline Junaeb & 1964 & Ministerio de Educación & $\begin{array}{l}\text { Acompañar a los/as estudiantes en condición de desventaja social, } \\
\text { económica, psicológica y/o biológica, para contribuir a la igualdad de } \\
\text { oportunidades dentro del sistema educacional, a través de la entrega } \\
\text { oportuna de bienes y/o servicios. }\end{array}$ \\
\hline Abriendo Caminos & 2008 & $\begin{array}{l}\text { Ministerio de Desarrollo } \\
\text { Social }\end{array}$ & $\begin{array}{l}\text { Apoyar a estos niños, niñas y adolescentes de } 0 \text { a } 18 \text { años, buscando } \\
\text { mitigar los efectos psicológicos, familiares y sociales que les provoca la } \\
\text { separación forzosa prolongada del adulto privado de libertad. }\end{array}$ \\
\hline
\end{tabular}

Elaboración propia. 
Una forma de abordar estos desafíos de protección de la vida y desarrollo de NNA en contextos de desigualdad social es lo que se propone en los 25 derechos definidos por el Consejo Nacional de la Infancia, emanados a su vez en la discusión en torno a la Convención sobre los Derechos del Niño (CDN) llevada a cabo por Naciones Unidas el año $2015^{16}$. La Convención sobre los Derechos del Niño (CDN) formaliza siete tipos de derechos de NNA: derechos a nivel de vida adecuado, a educación, a salud, a libertad de información y libertad de opinión, a la participación, a la protección en el trabajo, y a la vida y el desarrollo. Uno de los derechos clave que presente de manera transversal en los enunciados anteriormente es el de bienestar de NNA. Este derecho debiera estar presente desde la vida fetal y primera infancia de NNA, toda vez que lo que sucede durante estos primeros años de vida tiene efectos a lo largo de todo el curso de vida de las personas ${ }^{17}$. De esta manera, en un contexto global marcado por profundas desigualdades sociales entre países y dentro de ellos, la atención a la salud y el bienestar de la niñez se consideran un indicador clave del progreso en el desarrollo humano y económico, la igualdad y los derechos humanos.

Este derecho transversal de bienestar infantil, tal y como lo señalan Alfaro y colaboradores ${ }^{18}$, es esencial y requiere de mayor consideración en materia de política pública y protección de derechos de NNA. El campo conceptual del bienestar integra tres nociones y perspectivas de desarrollo relativamente paralelas, vinculadas a los conceptos de bienestar psicológico, bienestar subjetivo y bienestar social ${ }^{19}$. El bienestar subjetivo en particular se define como un componente psicosocial de la calidad de vida de los NNA, y percepciones que tienen respecto de sus condiciones vitales -tanto eco- nómico, como social, cultural, de salud y logros personales ${ }^{20}$. Incluye las dimensiones de afecto positivo, afecto negativo y satisfacción con la vida, en donde la visión predominante es la experiencia de placer frente al displacer, incluyendo los juicios sobre los buenos y malos elementos de la vida. Alfaro ${ }^{18}$ ha descrito algunos instrumentos de medición del bienestar subjetivo (tabla 3), entre ellos: (i) la Escala de Satisfacción con la Vida para Estudiantes (Student Life Satisfaction Scale, SLSS) creada por Huebner, (ii) la Escala Breve Multidimensional de Satisfacción con la Vida para Estudiantes (Brief Multidimensional Student Life satisfaction Scale, BMLSS) creada por Seligson, Huebner y Valois ${ }^{21}$; (iii) y el Índice de Bienestar Personal para Escolares (Personal Well-being Index- School Children, PWISC) creado por Cummins y $\mathrm{Lau}^{22}$. De acuerdo con un estudio realizado en Chile en el año 2015, aplicando estos instrumentos en población infantil y adolescente por el mismo autor, se evidenció que niños y niñas chilenos, con un alto Indice de Vulnerabilidad Escolar (IVE) y que asisten a colegios municipales, presentaron un bajo índice de satisfacción con la vida para cursos de séptimo básico comparado con los de quinto básico. Al comparar estos resultados con la evidencia internacional, se observa que, por ejemplo, el indicador de felicidad en NNA chilenos es menor al promedio internacional, estando por debajo de países como Argelia, Brasil, EEUU, Israel y Rumania ${ }^{13}$.

Sería beneficiosa la inclusión del bienestar subjetivo en la infancia en la estimación de gradientes sociales de salud poblacional. Estas gradientes son indicadores necesarios del acceso de los NNA a oportunidades justas, positivas y sistemáticas para su máximo desarrollo y florecimiento en todo el mundo. Esto porque la noción de bienestar subjetivo permite conocer y estimar

Tabla 3. Algunos de los instrumentos que miden el bienestar subjetivo de Niños y Niñas y Adolescentes utilizados en Chile

Escala de Satisfacción con la Vida para Estudiantes.

- Huebner (1991)

- Medir la satisfacción global con la vida de niños y niñas y adolescentes entre 8 y 18 años, a través del autoreporte de la satisfacción con la familia, amigos, colegio, barrio y la vida en general.

Escala Breve Multidimensional de Satisfacción con la Vida para Estudiantes

- Seligson, Huebner y Valois (2003)

- Medir la satisfacción global con la vida de niños y niñas y adolescentes entre 8 y 18 años al igual que la encuesta anterior, pero con menos preguntas para la eficiencia en su aplicación

Índice del Bienestar Personal

- Cummis y Lau (2008)

- Medir la satisfacción con la vida, desde el bienestar subjetivo y la calidad de vida en los aspectos más bien personales, lo que incluye posesiones materiales, la seguridad y la salud, necesidades básicas y diferentes aspectos del si mismo, como la autoestima, valores y las relaciones con otros

Elaboración propia 
la calidad de vida en un país o categoría social particular y, por tanto, orienta al diseño de acciones tendientes al logro de ésta. Esta información contribuye a validar empíricamente las condiciones necesarias para el "buen vivir", aportando en forma directa y práctica a reflexiones actuales en Chile, como por ejemplo qué es una "buena sociedad" y cómo alcanzarla, desde la propia voz de nuestros NNA. Propuestas concretas de utilización del concepto de bienestar subjetivo en salud de NNA podrían ser en la incorporación de su medición en control de niño sano, espacios amigables, niños con condiciones crónicas de salud y con situación de hospitalización crónica, encuestas poblacionales de salud, entre otras. También en investigación científica, que permita salir del enfoque de enfermedad/malestar y desarrolle mayor conocimiento desde las dimensiones de bienestar. De esta forma, la consideración del marco conceptual de bienestar subjetivo en la infancia y adolescencia tiene el potencial de complementar, aportar y fortalecer intervenciones de todo tipo que se realicen en NNA con enfoque de derechos y protección al bien superior de estos ${ }^{23}$.

En conclusión, el bienestar de los NNA es una base primordial para integrar en políticas públicas de infan- cia y adolescencia. Junto con esto, fortalecer y aumentar el número de estrategias de protección para ellos y ellas, surge como un imperativo en respuesta a aquellos grupos de NNA que viven en desventaja social tanto en Chile como en el resto del mundo. Se propone, por lo tanto, incorporar de manera formal y explícita el concepto de bienestar subjetivo en los objetivos de los planes de protección a los NNA, donde no solo los tomadores de decisión gubernamentales conozcan esta herramienta y entiendan su complejidad, sino también los profesionales que trabajan día a día con ellos y ellas como profesores, personal de la salud, entre otros. Metas de aseguramiento del bienestar en general, y del bienestar subjetivo en particular, en NNA facilitará el desarrollo sano y feliz de cada uno de ellos a lo largo de todo su ciclo de vida, con especial énfasis en aquellos que sufren las consecuencias de las desigualdades sociales estructurales que como sociedad hemos construido.

\section{Conflicto de intereses}

Las autoras declaran no tener conflicto de intereses.

\section{Referencias}

1. Ministerio de Desarrollo Social G de C. Encuesta Caracterización Socioeconómica Nacional. 2018; Available from: http:// observatorio.ministeriodesarrollosocial. gob.cl/casen-multidimensional/casen/ docs/Resultados_pobreza_Casen_2017. pdf

2. Ministerio de Relaciones Exteriores. Decreto $n^{\circ}$ 830: Promulgación de los derechos del niño [Internet]. Biblioteca Congreso Nacional. 1990. Available from: http://bcn.cl/luvqj

3. Fondo de las Naciones Unidas para la Infancia. Estado mundial de la infancia 2016: Una oportunidad para cada niño. 2016. 1-73 p.

4. Unicef. Agenda de infancia 2018-2021: Desafíos en un área clave para el país. 2018.

5. MIDEPLAN. Principales Resultados Encuesta de Actividades de Niños, Niñas y Adolescentes (EANNA) 2012. 2013;1-33. Available from: http:// observatorio.ministeriodesarrollosocial. gob.cl/layout/doc/eanna/presentacion EANNA_28junio_final.pdf

6. Gunnar M, Quevedo K. The Neurobiology of Stress and Development. Annu Rev Psychol. 2007;58:145-73.

7. Hair NL, Hanson JL, Wolfe BL, Pollak SD. Association of Child
Poverty, Brain Development, and Academic Achievement. JAMA

Pediatr [Internet]. 2015;169(9):822-9. Available from: https://doi.org/10.1001/ jamapediatrics.2015.1475

8. Reynolds AJ, Temple JA, Ou S-R, et al. Effects of a School-Based, Early Childhood Intervention on Adult Health and Well-being: A 19-Year Follow-up of Low-Income Families. JAMA Pediatr [Internet]. 2007;161(8):730-9.

9. Franke HA. Toxic Stress: Effects, Prevention and Treatment. 2014 2014;1(3):390-402. doi: 10.3390/ children 1030390.

10. Sánchez MM, Ladd CO, Plotsky PM. Early adverse experience as a developmental risk factor for later psychopathology: evidence from rodent and primate models. Dev Psychopathol. 2001;13(3):419-49.

11. McCrory E, De Brito SA, Viding E. The impact of childhood maltreatment: a review of neurobiological and genetic factors. 2011. 28;2:48. doi: 10.3389/ fpsyt.2011.00048

12. Ministerio Secretaría General de la Presidencia. Política Nacional de Niñez y Adolescencia 2015-2025. Versión para niños, niñas y adolescentes. 2015;1-38.

13. Programa de las Naciones Unidas para el Desarrollo (PNUD). Índices E
Indicadores De Desarrollo Humano. Programa las Naciones Unidas para el Desarro. 2018;1-123.

14. Fondo de las Naciones Unidas para la Infancia. Una oportunidad justa para cada niño Documento de conferencia. Sesión Junta Ejecutiva UNICEF dedicada a la Equidad. 2015.

15. Equalitytrust.org.uk. (2019). Child Well-being | The Equality Trust. [online] Available: https://www.equalitytrust.org. uk/child-well-being [Accessed 13 Sep. 2019].

16. Organizacion de las Naciones Unidas. Asamblea general ONU: Convención sobre los Derechos del Niño. [Internet]. 2015; Available from: www.unicef.es

17. Alfaro J, Casas F, López V. Bienestar en la infancia y adolescencia. Psicoperspectivas [Internet]. 2015. Available from: https://scielo.conicyt.cl/ scielo.php?script=sci_arttext\&pid $=$ S071869242015000100001\&lng=es\&nrm=iso\&t lng $=$ es.

18. Alfaro JGJ, Oyarzún D, Reyes F, Sirlopú D, Varela J. Bienestar subjetivo de la infancia en Chile en el contexto internacional. Santiago, Chile: Publicaciones psicología UDD; 2015.

19. Blanco A, Valera S. "Los fundamentos de la intervención psicosocial”. En: Amalio B \& Rodríguez J. Intervención Psicosocial. Madrid: 2007. McGraw-Hill. 
20. Casas F, Fernández-Artamendi S, Montserrat C, Bravo A, Bertrán I, Valle JF. El bienestar subjetivo en la adolescencia: estudio comparativo de dos Comunidades Autónomas en España. Anal. Psicol. [Internet]. 2013; 29(1):14858. Disponible en: http://scielo.isciii.es/ scielo.php?script=sci_arttext\&pid=S021297282013000100018\&lng=es. [citado el 9 de septiembre de 2019].

21. Huebner ES, Suldo SM \& RFV.

Psychometric Properties of Two Brief

Measures of Children's Life Satisfaction:

The Students' Life Satisfaction Scale

(SLSS) and the Brief Multidimensional Students' Life Satisfaction Scale

(BMSLSS). Indic Posit Dev Conf. 2003; 1997:1-49.
22. Cummins RA, Lau ALD. Personal Wellbeing Index-Intellectual Disability (English) 3 rd Edition The International Wellbeing Group. 2005;

23. Tisdall E. Derechos y bienestar de los niños: ¿conceptos de política equivalentes? Revista de Política Social. Prensa de la Universidad de Cambridge; 201544 (4): 807-23. 